\title{
Vestibular and non-vestibular contributions to eye movements that compensate for head rotations during viewing of near targets
}

\author{
Yaming H. $\operatorname{Han}^{1}$ \\ Arun N. Kumar ${ }^{1}$ \\ MillardF. Reschke $e^{3}$ \\ Jeffrey T. Somers ${ }^{3}$ \\ Louis F. Dell'Osso ${ }^{1,2}$ \\ R. John Leigh ${ }^{1,2}$
}

${ }^{1}$ Departments of Biomedical Engineering, and ${ }^{2}$ Neurology,

Department of Veterans Affairs Medical Center and University Hospitals,

Case Western Reserve University, Cleveland, Ohio 44106, U.S.A.

${ }^{3}$ Neuroscience Laboratory, NASA, Johnson Space Center, Houston, Texas

Running heading: VOR during near viewing

Address Corresp ondence to:

R. John Leigh, M. D., Department of Neurology, University Hosp itals,

11100 Eudid Avenue, Cleveland, Ohio, 44106-5040. 
Phone: (216)-421-3224; FAX:(216)-231-3461; E-mail: rj4@po.cwru.edu

\section{Abstract}

We studied horizontal eye movements induced by en-bloc yaw rotation, over a frequency range $0.2-2.8 \mathrm{~Hz}$, in 10 nomal human subjects as they monocularly viewed a target located at their near point of focus. We measured gain and phase relationships between eye-in-head velocity and head velocity when the near target was either earth-fixed or head-fixed. During viewing of the earth-fixed near target, median gain was 1.49 (range $1.24-1.87$ ) at $0.2 \mathrm{~Hz}$ for the group of subjects, but declined at higher frequencies, so that at $2.8 \mathrm{~Hz}$ median gain was 1.08 (range $0.68-1.67$ ). During viewing of the head-fixed near target, median gain was 0.03 (range $0.01-0.10)$ at $0.2 \mathrm{~Hz}$ for the group of subjects, but increased at higher frequencies, so that at $2.8 \mathrm{~Hz}$ median gain was 0.71 (range $0.28-0.94$ ). We estimated the vestibular contribution to these resp onses (vestibulo-ocular reflex gain, Gvor) by applying transient head perturbations ( eak acceleration $>1,000 \mathrm{deg} \mathrm{s}^{2}$ ) during sinusoidal rotation under the two viewing conditions. Median Gvor, estimated $<70 \mathrm{~ms}$ after the onset of head perturbation, was 0.98 (range 0.39 1.42) while viewing the earth-fixed near target, and 0.97 (range $0.37-1.33$ ) while viewing the head-fixed near target. For the group of subjects, 9 out of 10 subjects showed no significant difference of Gvor between the two viewing conditions $(\mathrm{p}>0.053)$ at all test frequencies. Since Gvor accounted for only $\sim 73 \%$ of the overall response gain during viewing of the earthfixed target, we investigated the relative contributions of non-vestibular factors. When subjects viewed the earth-fixed target under strobe illumination, to elminate retinal image slip information, the gain of compensatory eye movements declined compared with viewing in ambient room light. During sum-of-sine head rotations, while viewing the earth-fixed target, to 
Han et al., /VOR during near-viewing

minimize contributions from predictive mechanisms, gain also dedined. Nonetheless, simple superposition of smooth-pursuit tracking of sinusoidal target motion could not fully account for the overall response at higher frequencies, suggesting other non-vestibular contributions. During binocular viewing conditions when vergence angle was significantly greater than monocular viewing $(p<0.001)$, the gain of compensatory eye movements did not show prop ortional change; indeed, gain could not be correlated with vergence angle during monocular or binocular viewing. We conclude that several separate factors contribute to generate eye rotations during sinusoidal yaw head rotations while viewing a near target; these include the VOR, visual-tracking eye movements that utilize retinal image motion, predictive eye movements and, possibly, other unidentified non-vestibular factors. For these experiments, vergence was not an important determinant of resp onse gain.

\section{INT RODUCTION}

In order to see the environment clearly, images of stationary objects must be held fairly still on the retina (Carpenter 1991). During natural activities, especially locomotion, head perturbations with predominant frequencies ranging up to $5 \mathrm{~Hz}$ pose a threat to clear vision (Grossman et al. 1988; Das et al. 1995b; Crane and Demer 1997a). The vestibulo-ocular reflex (VOR) generates eye rotations at short latency $(<15 \mathrm{~ms})$ that can compensate for such head perturbations (Maas et al. 1989; Collewijn and Smeets 2000) and maintain a clear and stable visual percept. Individuals who have lost vestibular function report that they cannot see their environment clearly while they are in motion (J.C. 1952). Thus, the VOR is indispensable for 
Han et al., /VOR during near-viewing

clear vision during natural activities (Leigh and Zee 1999). Nonetheless, other factors contribute to generate eye rotations that compensate for head perturbations. One factor is visually mediated eye movements, such as smooth pursuit, that can compensate for head movements, but act at longer latencies $(\sim 100 \mathrm{msec})$ than the VOR (Carl and Gellman 1987). Another factor is the predictive mechanism that acts to negate the delay inherent in visual signal processing (Dallos and Jones 1963; Bames 1993; Bames et al 2000). The contribution of non-vestibular factors can be demonstrated, for example, when subjects attempt to fixate the remembered location of a target in darkness during head rotations, the compensatory response is less than if they actually view the visual tanget (Barr et al. 1976; Das et al. 2000). In this paper, we refer to the sum of vestibular and non-vestibular factors contributing to eye movements that compensate for head rotations as the enhanced VOR (EVOR). How much non-vestibular factors contribute to EVOR ap pears to vary acoording to species and test paradigms.

Special demands are made when subjects view a near, earth-fixed stationary target during head rotations (Blakemore and Donaghy 1980; Bigner and Prablanc 1981; Virre et al. 1986; Hine and Thorn 1987; Han et al. 2001). Since the eyes do not lie on the axis of head rotation, they are displaced (translated) as well as rotated when the head tums. Thus, during the near-viewing situation, in order to hold the gaze on the target, the eye must rotate more than the head; with the target at the subject's near point of accommodation the gain of EVOR (eye velocity / head velocity) can exced 1.5. Prior studies in monkey (Vïre et al. 1986; Snyder and King 1992) have suggested that such gain increases, which ocaur within $20 \mathrm{~ms}$ of the onset of ey e movements, are due to an increment of the internal gain of the VOR (Gvor). In humans, 
Han et al., /VOR during near-viewing

the gain of EVOR during viewing of a near target may occur even earlier (8-18 ms) after the onset of head rotation(Crane and Demer 1998). The questions addressed in the present study were: (1) How much does Gvor change during viewing of a near target? (2) what non-vestibular factors contribute to EVOR? (3) What is the behavior if subjects view a near target that is headfixed (rather than earth-fixed)? Under this last condition, the VOR may be canceled by visualtracking mechanisms, such as smooth pursuit (Cullen et al. 1991; Huebner et al. 1992). Preliminary results have been published as short reports (Han et al. 2003, 2005).

\section{ETHODS}

\section{Subjects}

We studied 10 nomal subjects ( 4 female, age $24-57$ y ear). Five subjects were naive as to the purpose of the study, two were experienced in ocular motor studies, and three were aware of the purpose of the experiments. No subjects had any ocular motor abnormalities or were taking drugs with effects on the nervous system. Five subjects were my opes (corrections were $-3.5 \mathrm{D}$ on average), but were able to clearly see the near visual stimuli without their spectacle corrections throughout the testing. All subjects gave written, informed consent in accordance with our Institutional Review Board and the tenets of the Dedaration of Helsinki

\section{Experimental stimuli}

Head and ey e rotations were measured using the magnetic search coil technique, with 6$\mathrm{ft}(1.8 \mathrm{~m})$ field coils (CNC Engineering, Seattle, WA) that used a rotating magnetic field in the 
Han et al., /VOR during near-viewing

horizontal plane and an alternating magnetic field in the vertical plane. Each subject wore a pair of scleral search coils (Skalar Delft, Netherlands) on both ey es to measure gaze ange, and a third coil firmly attached on the forehead to measure angular head rotations. Search coils were calibrated on a protractor device before experiments. The system was $98.5 \%$ linear over an operating range of $\pm 20 \mathrm{E}$ in both horizontal and vertical planes, and the SD of system noise was $<0.02 \mathrm{E}$.

Angular head rotations in yaw were applied using a vestibular chair (Templin Engineering, Laytonville, Calif). Subjects sat in a $30-\mathrm{ft}-\mathrm{lb}$ vestibular (motor driven) chair in the search coil field, with their heads aligned so that the axis of chair rotations corresponded to the axis of their natural head rotations (close to the mid-interaural point). They wore a modified cycle helmet that contained foam pads to ensure a snug fit for each subject. During the experiment, subjects braced their heads against the headrest of the chair through out the recording session.

Five types of visual stimuli were used in these experiments:(1) A far target consisting of a red laser spot subtending an angle of $0.05 \mathrm{E}$ projected onto a wall at a viewing distance of 3 $\mathrm{m}$; it was either viewed in ambient light, or flashed at ap proximately $1 \mathrm{~Hz}$ in an otherwise dark room. (2) An earth-fixed near target, consisting of a black cross $\left(1 \mathrm{~cm} \_1 \mathrm{~cm}\right)$ drawn on a small piece of white tape was attached to a piece of wood, positioned at the ey e level of each subject. With one of the subject's ey e patched throughout the experiment, the cross target was aligned on the viewing ey e at a distance of $\sim 12 \mathrm{~cm}$, corresponding to the near point of accommodation for each subject. (3) A head-fixed near target, consisting of a similar black cross attached to a 
Han et al., /VOR during near-viewing

rigid plastic rod attached to the modified cycle hemet; the target was aligned on the viewing eye, at a similar distance to the earth-fixed near target. The main experiments were conducted in ambient room light. (4) In control experiments, we used strobe illumination, while four subjects viewed the earth-fixed near target. A flash rate of $4 \mathrm{~Hz}$, with $30 \mathrm{Fs}$ flash duration, was used, which has been used previously to eliminate retinal image slip during vestibular experiments (Melvill Jones and M andl 1981). As a control experiment, we repeated these experiments with a flash frequency of 3 times the rotational head frequency and the same flash duration; in this way the same amount of target position information was available during each rotational cycle. (5) A near, horizontal smooth-pursuit target, consisting of a red laser spot (subtending an ange of $0.7 \mathrm{E}$ ) was projected onto the black surface of a horizontally positioned board, which lay just below the subject's ey e level. The zero-position of target was adjusted so that it was aligned on the viewing eye at the same distance $(\sim 12 \mathrm{~cm})$ as the earth-fixed and head-fixed near targets. The position of the moving target was under computer control, using a $\mathrm{X}-\mathrm{Y}$ mirror galvanometer (model CCX-660, General Scanning Watertown, Mass). Pursuit experiments were performed in an otherwise darkened room and subjects' heads were held still through out the testing.

Vestibular stimuli consisted of three types of en bloc y aw rotations: (1)_Sinusoidal head rotations: at each of five test frequencies $-0.2,0.7,1.0,2.0$, and $2.8 \mathrm{~Hz}$ with a constant peak velocity of $15 \mathrm{deg}$ sec. At the beginning of each session, each subject was rotated at a frequency of $0.1 \mathrm{~Hz}$ with the peak velocity of $15 \mathrm{deg} / \mathrm{sec}$ while they monocularly viewed the constantly illuminated stationary laser far target. This procedure served as a calibration check 
Han et al., /VOR during near-viewing

and assumed that our subjects continuously foveated on the target and generated compensatory eye movements with a gain of 1.0. A second calibration check was made as each subject was rotated at $0.1 \mathrm{~Hz}$ with peak velocity of $15 \mathrm{degsec}$ during continuously viewing of the headfixed near target; assuming continuous foveation of this target, no ey e-in-head rotations should be generated. (2) Transient head perturbations: in order to measure dy namic internal VOR level (gain - Gvor) and to quantify the vestibular contributions to the overall ocular responses under different viewing conditions, we applied transient head perturbations during sinusoidal rotations when subjects were viewing an earth-fixed or head-fixed near target at the five test frequencies. Since the velocity of vestibular chair was controlled by a voltage signal (motor servo), we used a computer program that generated the accleration pulses by changing the sign (representing the direction of chair rotation, left or right) of the control signal at the peak velocity. Consequently, at each perturbation the chair velocity changed from $15 \mathrm{deg}$ s to $15 \mathrm{deg} / \mathrm{s}$ abruptly, or viœ versa, generating a peak accleration $>1,000 \mathrm{deg} / \mathrm{s} / \mathrm{s}$; there were a total of nine perturbations in each 40 s trial. (3) Sum-of-sines stimuli (pseudorandomrotations): in order to investigate the contribution to the responses of predictable visual tracking eye movements, we applied sum-of-sines stimuli (pseudorandom chair rotations) in 4 subjects during viewing of the earth-fixed near target. The component sine waves had frequencies of $0.38 \mathrm{~Hz}, 1.23 \mathrm{~Hz}, 2.08 \mathrm{~Hz}$ and $2.63 \mathrm{~Hz}$, with peak velocities of $3.3,5.5,6.0$, and $15.3 \mathrm{deg} / \mathrm{sec}$, respectively. Thus, the velocity ratio between the highest and lowest frequency components was $>4.0$, which Bames (1993) has established as a reliable strategy for preventing predictive visual tracking 
Han et al., /VOR during near-viewing

Experimental paradigms

There were five main experiments (conducted in five sessions, in which 10 subjects participated) and four control experiments (conducted in three sessions, in which four subjects participated). Each trial lasted $40 \mathrm{sec}$. Subjects were instructed to maintain fixation of the near stimuli using one ey (chosen by themselves); the other eye was occluded but its position was monitored by an eye coil during Main Experiments 1 and 2. For the other experiments, only the viewing eye was wearing an eye coil, except for the vergence control experiment that required binocular viewing.

\section{Main Experiments:}

(1) Head rotations in darkness (VOR): Subjects were asked to attempt to fixate the remembered location of the flashing far target while they were rotated sinusoidally in the vestibular chair in darkness at the five test frequencies.

(2) Head rotations viewing the earth-fixed near target (EVOR): Subjects were rotated at each of the five test frequencies under ambient room light illumination.

(3) Head rotations viewing the head-fixed near target (eye-head tracking EHT): Subjects were rotated at each of the five test test frequencies under ambient room light illumination.

(4) Head perturbations during EVOR and EHT: Paradigms 2 and 3 were repeated, during which transient head accelerations were delivered.

(5) Smooth pursuit: Subjects were asked to track the near smooth-pursuit target at each of the five test frequencies in an otherwise darkened room. 
Han et al,, /VOR during near-viewing

\section{Control Experiments:}

(6) EVOR under strobe illumination: Paradigm 2 was repeated but under stroboscopic illumination when the flash rate was fixed at $4 \mathrm{~Hz}$ for the frequency range of head rotations. In two subjects, paradigm 2 was repeated with a flash rate adjusted to 3 times the rotational head frequency.

(7) EVOR during sum-of-sines rotation: Paradigm 2 was repeated using the sum-of sines stimulus.

(8) EVOR and EHT during binocular viewing: Paradigms 2 and 3 were repeated as five subjects viewed the near targets binocularly (aligned on the eye that had viewed monocularly). We positioned the near targets at the same distance during binocular or monocular viewing, so that we were able to compare how overall resp onse gain changed when vergence angle differed.

\section{Data collection and analysis}

Horizontal and vertical head and gaze (eye-in-space) signals were lowpass filtered using Krohn-Hite Butterworth filters with a bandwidth of $0-150 \mathrm{~Hz}$, prior to digitization with 16 bit precision at $465 \mathrm{~Hz}$ or $500 \mathrm{~Hz}$. For sinusoidal head stimulations, head and gaze signals were nomalized for each subject according to their response to the $0.1 \mathrm{~Hz}$ stimulus, based on the assumption they could continuously foveate the visual target. For near smooth pursuit, the target signal at each frequency was normalized for each subject as they followed a $0.2 \mathrm{~Hz}$ square wave that stepped a similar amplitude to the sinusoidal stimulus that followed. Portions of data contaminated by blinks or any extraneous saccades were visually identified and 
Han et al., /VOR during near-viewing

discarded. Eye-in-head rotations (referred to, hereafter as "eye position") were calculated by subtracting the head position signal from eye-in-space (gaze) signal. Convergence ange was obtained by subtracting right gaze from left gaze. We differentiated these signals to obtain corresponding velocity measurements, and filtered these signals with a Remez filter (bandwidth 0-40 Hz) (Ramat et al. 1999). Prior to filtering, saccades were removed from the eye and gaze signals via an interactive routine described elsewhere (Das et al. 1995a). We determined the gain of EVOR during head rotations for each trial by calculating the ratio of power spectral density of eye and head velocities at the frequencies of interest, including the sum-of-sines stimuli, using a fast Fourier transform ( FFT) method. EHT gain during viewing of a head-fixed near target and smooth pursuit gain during tracking of a near target were calculated by taking the ratio of power spectral density of gaze and target velocities at the frequencies of interest. The phase response was determined by measuring phase angle of these paired signals and recording the phase difference between them at the frequency of interest.

For transient head perturbation stimuli, we defined stimulus onset as when head acceleration exceeded $200 \mathrm{deg} / \mathrm{s} / \mathrm{s}$, and measured "onset head velocity" and "onset ey e velocity" at this point. We then determined, interactively, the peak head and ey e velocity values in the $70 \mathrm{~ms}$ epoch following the stimulus onset (Aw et al. 1996). The value of Gvor for each response was calculated by equation (1):

There were a total of nine head perturbations employed at each frequency of head 
Han et al., NOR during near-viewing

rotation, and from these responses we calculated the median value of Gvor for each test condition. As a check of the reliability of this methodology, we also measured the median value of ey e and head velocity in the $70 \mathrm{~ms}$ following stimulus onset, and used these values to make a separate estimate of Gvor from equation (2):

Similar results were obtained, and here we present estimates of Gvor based on measurements by equation (1). Paired statistical comparison were made between resp onses at each frequency and for each subject by either a paired t-test or, if the distribution of data were not normal, using a Wikoxon rank-sum test.

\section{RESULT S}

General features of responses to simisoidal rotation

Representative responses from one subject during the experimental conditions 1-3 are shown in Figure 1. Responses during rotation in darkness while viewing a flashing far target are shown in Figure 1A and B; at $0.2 \mathrm{~Hz}$ gain is 1.0 and at $2.0 \mathrm{~Hz}$ it is 0.9 . During viewing of the earth-fixed near target, at $0.2 \mathrm{~Hz}(\mathrm{C})$, the subject was able to maintain continuous foveation of the near target, and EVOR gain was 1.49 . At $2.0 \mathrm{~Hz}$ (D), gain declined to 1.37; thus, at this higher frequency of head rotation, ey e movements did not fully compensate for head rotations. During viewing of the head-fixed target, at $0.2 \mathrm{~Hz}(\mathrm{E})$, the EVOR gain was 0.01 , close to an 
Han et al., /VOR during near-viewing

ideal gain of zero for this viewing situation. At $2.0 \mathrm{~Hz}(\mathrm{~F})$, gain increased to 0.39 , indicating that the VOR was not completely suppressed or canceled.

Figure 2 summarizes the gain values of responses from ten subjects as they viewed the far target flashed in darkness, the earth-fixed near target, or the head-fixed near target. Note that values for the head-fixed viewing condition (Figure $2 \mathrm{E}$ and $\mathrm{F}$ ) are plotted as tracking gain (corresponding to 1-EVOR gaim), so that these responses can be compared directly with smooth-pursuit behavior, which is described later. At lower frequencies of rotation, the gain of compensat ory responses is over $50 \%$ greater during viewing of the earth-fixed near target (Figure 2C) than the flashed far target in darkness (Figure 2A). However, at higher frequencies of head rotation, the gain and phase shift of EVOR declined during viewinig of the earth-fixed near target, but changed little for the flashed far target in darkness. During viewing of the near head-fixed target, tracking gain declined and phase lags increased for rotational frquencies above $1 \mathrm{~Hz}$.

Head Perturbations: Determination of the vestibular contribution to the responses

Figure 3A and B provide examples of responses to head perturbations as subjects are sinusoidally oscillated while viewing an earth-fixed or head-fixed near target. The value of Gvor for these two responses was similar. Figure 4 summarizes median values for ten subjects, for each of the five frequencies, that are plotted as frequency histogams. For comparison, we also plot measurements of Gvor during rotation in darkness (Figure $4 \mathrm{C}$ ); note that for $\mathrm{A}$ and $\mathrm{B}$, Gvor values are dynamic VOR gains measured through transient head perturbations during 
Han et al., /VOR during near-viewing

viewing of a near target, while Gvor in Figure $4 \mathrm{C}$ are steady state gain values during attempting to fixate a remembered far tanget in darkness. The range of values was similar for the two neartarget visual stimuli, but Gvor values were smaller when tested in darkness. Substantial variance of data is evident in these histograms, simlar to prior studies (Collewijn and Smœts 2000). In general, lower values of Gvor were in my opes who wore spectacle corrections, which is a reoognized association (Cannon et al. 1985).

We carried out paired t-tests comparing for each subject and frequency, median gain values for the earth-fixed and the head-fixed target viewing conditions (F igure 4D); there was no significant difference in 9 out of the 10 subjects $(p>0.053)$. One subject showed a significant greater value of Gvor during viewing of the earth-fixed target than viewing of the head-fixed target $(\mathrm{p}<0.001)$.

We also compared Gvor values based on transient head perturbations during viewing of an earth-fixed near target with Gvor measured in darkness; for the group of subjects, a paired ttest showed that transient perturbation Gvor was significantly greater than Gvor measured in darkness for the group of subjects $(p=0.007)$. The median value of Gvor during viewing of an earth-fixed target was 0.98 for the group of subjects (range $0.39-1.42$ ), whereas the median value of Gvor measured in darkness was 0.91 (range 0.54 - 1.04). However, when we compared Gvor values based on transient perturbations during viewing of a head-fixed target with Gvor measured in darkness, a paired t-test showed no significant difference for the group of subjects $(p=0.078)$. The median value of Gvor during viewing of a head-fixed target was 0.97 for the group of subjects (range $0.37-1.33$ ). 
Han et al., /VOR during near-viewing

We made an estimate of the percentage contribution of Gvor to the gain of EVOR during viewing of the earth-fixed near target (Figure 5). Overall, Gvor only accounted for $73 \%$ of the response, less for greater response gains, indicating non-vestibular factors are critical in modulating VOR gain to an appropriate level.

Control Experiments: Identification of non-vestibular factors

Since Gvor did not fully account for the observed behavior, in the control experiments we tested four potential factors that could contribute to EVOR gain: retinal image motion; effects of the predictive nature of the stimulus motion; properties of visual tracking eye movements; and vergence angle.

\section{Contribution of retinal slip to the non-vestibular component of the EVOR}

In four subjects, we measured the gain of EVOR during viewing of the near earth-fixed target under strobe illumination at $4 \mathrm{~Hz}$ with 30 microseconds flash duration, which essentially abolished retinal slip. In Figure 6 , gains during nomal illumination were plotted as black symbols connected by black lines; gains during strobe illumination were plotted in open sy mbols connected by dotted lines. The results indicated, how, especially at frequencies of head rotation of $\$ 1.0 \mathrm{~Hz}$, each of the four subjects showed higher EVOR gain during nomal illumination (when retinal slip information was available) compared to strobe illumination. However, strobe illumination also provides visual cues during each flash, and this result could be explained by the fact that more position cues occurred at lower compared with higher 
Han et al., /VOR during near-viewing

rotational frequencies. To test this possibility, in two subjects, we repeated the experiment using a strobe rate which was always three times the frequency of head rotation. In this way, the number of position cues occurred in each cy cle was constant for all stimulus frequencies. In Figure 6, these data are plotted as gray symbols connected by gray lines. Both subjects generally showed lower gain values during this strobe stimulus than under ambient illumination. Thus, irrespective of flash frequency, gain was reduced, suggesting that retinal image velocity is used by the brain to optimize the performance of compensatory eye movements, including the higher frequencies of head rotation that we employ ed.

\section{Contribution ofprediction to the non-vestibular component of the EVOR}

We employ ed pseudorandom (sum-of-sines) head rotations in 4 subjects to minimize contributions of predictive eye movements during viewing of the earth-fixed near target. Results were compared with responses to head rotations at each component frequency of sumof-sines. Figure 7 showed comparison of EVOR gain between sum-of-sines and sinusoidal head rotations at each of the component frequencies. All four subjects showed greater values of the gain of compensatory eye movements for single sine-wave stimuli than for corresponding components of the sum-of-sines stimulus; this was esepcially true for lower frequencies. These results support the view that predictive mechanisms contribute to the overall EVOR.

\section{Comparison of smooth pursuit and the non-vestibular component of the EVOR}

Since retinal image motion and prediction both appear to contribute to the non- 
Han et al., /VOR during near-viewing

vestibular component, we formally tested whether visual tracking eye movements, specifically smooth pursuit, could completely account for the non-vestibular contribution that we identified during viewing of the earth-fixed near target. We measured smooth-pursuit responses to a near target moving at same frequencies and velocities as the chair movements that we employed during viewing of the near, earth-fixed target (EVOR). We used a superposition model (Figure 8) to describe the interactions between VOR and non-VOR in accounting for the general behavior that we observed (Figures 1 and 2). Although this model does not incorporate elements to account for the dy namic characteristics of pursuit onset (Krauzlis and Lisberger 1994), we were concerned with steady-state responses to sinusoidal stimuli for both pursuit and EVOR, and assumed that predictive mechanisms would have similar influenœs under the two conditions. We first used a parameter estimation method implemented in the frequency domain to estimate the optimal values of the non-vestibular parameters (Das et al. 1998; Guild et al. 2001). The estimation procedure is summarized in the Appendix For each subject we set Gvor at the experimentally determined value during paradigm 4 (head perturbations during viewing an earth-fixed near target). Then we estimated optimal values for non-vestibular parameters of the model using the earth-fixed near-viewing EVOR responses at each frequency of head rotations. Model fits of subjects' responses were generally good, with residual values of $<10 \%$ and parameter coefficient of variations of $<12 \%$. The op timal values of the two nonvestibular parameters, for each subject and test frequency, are summarized in Table 1. Finally we compared non-vestibular model's predictions of visual tracking with measured smooth pursuit performance for each subject and frequency. In this way, we could determine if smooth 
Han et al., /VOR during near-viewing

pursuit accounted fully for the non-vestibular factor. Figure 9 compares model predictions and measured pursuit performance to a near target in gain (A) and phase shift (B). Model predictions deviated substantially from subjects' pursuit responses at high frequencies $(>1.0$ $\mathrm{Hz}$ ), with larger gain and smaller phase shift. A paired comparison of model versus experimental gain values (Figure 9A) showed substantial differences, especially at higher frequencies. This result indicated that smooth pursuit alone can not acoount for the nonvestibular contributions to the EVOR during viewing of the earth-fixed near target. We also compared the model's predictions of EHT during viewing of a head-fixed near target (Figure $2 \mathrm{E}$ and F), making paired comparisons of predicted and observed tracking gain for each frequency and each subjects. The model made much better predictions of gain for EHT (Figure 10B) than for smooth pursuit (Figure 10A), especially at higher test frequencies. Thus, the non-vestibular component differs from smooth pursuit but accounts better for EHT behavior while viewing a head-fixed target.

Experiments to determine if vergence angle is responsible for EVOR adjustment during near viewings

Our main experiments 2 and 3 were carried out as 10 subjects monocularly viewed either an earth-fixed or a head-fixed near target $(12 \mathrm{~cm})$, but were wearing two eye coils to monitor the vergence angle. For monocular viewing, the only stimuli to convergence are acoommodation and the perception of nearness for the viewing ey e; there is no visual feedback control of vergence. Figure $11 \mathrm{~A}$ and $\mathrm{B}$ plot the EVOR gain against vergence angle during 
Han et al., /VOR during near-viewing

monocular viewing at the two viewing conditions; no relationship between vergence angle and EVOR gain is apparent.

In addition, we repeated the experiments in 5 subjects during which subjects were viewing the earth-fixed or head-fixed near target binocularly. In this way, we were able to investigate how the gain of compensatory eye movements changed when vergence eye movements could be visually controlled. Figure $11 \mathrm{C}$ systematically compares the gain of compensatory responses for earth-fixed (EVOR) or head-fixed (EHT) near targets for either monocular or binocular viewing. The vertical dashed line draws the approximate boundary of vergence angle between monocular and binocular viewing.Thus, open sy mbols correspond to monocular viewing(left side of the vertical dashed line); gray sy mbols correspond to binocular viewing (right side of the vertical dashed line). The horizontal dashed line shows the approximate boundary of EVOR gain values during viewing an earth-fixed or a head-fixed target. Thus, sy mbols with thick edge (above the horizontal dashed line) are during viewing of an earth-fixed target; symbols with thin edge (below the horizontal dashed line) are during viewing of a head-fixed target. In general, binocular viewing was associated with greater vergence anges than monocular viewing, but there was no difference in response gain (EVOR or EHT) associated with binocular or monocular viewing. One subject was able to maintain the same vergence ange during monocular and binocular viewing at all test frequencies (data points enclosed by an ellipse indicated by arrows, close to the vertical dashed line on Figure 11C); despite this, his EVOR gain was significantly varied. Thus, our present results do not support the hypothesis that vergence angle determines the gain of eye movements to compensate for 
Han et al., /VOR during near-viewing

head rotations during viewing of a near target.

\section{I S C US SION}

We set out to understand better vestibular and non-vestibular mechanisms contributing to eye rotations that compensate for head rotations (EVOR), while viewing a near target. Under such viewing conditions, geometry dictates that ey e rotations exceed head rotations by $50 \%$ or more, depending mainly on the proximity of the target. Prior studies, especially those employ ing transient stimuli (Sny der and King 1992; Crane and Demer 1998), indicated that an increase of the gain of the VOR (Gvor) was largely responsible for eye rotations exceeding head rotations. Using sine-wave head rotations in humans, we confirmed that, at low frequencies of head rotation, the gain of EVOR was appropriately increased so as to maintain foveation of the earth-fixed near visual target (Figure 1C). However, all subjects showed a decline in gain and increased phase shift at higher rotational frequencies (Figure 2C and D). This result suggested to us that non-vestibular factors, such as visual tracking contributed to the lower frequency response but were unable to sustain their contribution at higher frequencies (Figure 9). By applying head perturbations during sinusoidal stimulation, we were able to confirm that modulation of Gvor could not solely account for the gain of compensatory eye movements during near-viewing (Figure 5). In control experiments, we went on to try and identify the relative contributions of vestibular and non-vestibular factors. We also investigated vestibular and non-vestibular factors when subjects viewed a near, head-fixed target, since the contribution of smooth pursuit to this eye-head tracking behavior has been previously 
Han et al., /VOR during near-viewing

investigated (Cullen et al. 1991; Huebner et al. 1992). In discussing these findings, first, we summarize current evidence for a vestibular contribution to the increased gain of compensatory eye movements during near-viewing response. Second we will review the possible role play ed by non-vestibular mechanisms, including visual tracking and predictive mechanisms. Third, we will examine the relationship between vergence angle and EVOR. Finally, we will discuss the relevance of our findings to performance ofEVOR during natural activities.

The vestibular contribution to generation of compensatory eye movements during near-viewing

Several studies have identified the geometric factors that determine the relationship between head rotations and ey e rotations that perfectly maintain the line of sight on a specified target (Blakemore and Donaghy et al. 1980; Biguer and Prablanc 1981; Vïrre et al. 1986). These studies also experimentally confirmed in cats, monkeys, and humans that target location, ey e-head geometry, and axis of head rotation are important determinants of the response. In the present study, we aligned near visual targets on the horizontal axis of one eye and attempted to rotate subjects' heads about an axis comesponding to their natural head movements; we did not set out to study the effects of eccentric head rotation.

Having defined the geometry and behavior, subsequent research has focused on the neural mechanism for the near-viewing resp onse to head rotation. In monkeys, Virre et al. (1986) demonstrated that the gain of compensatory eye movements during near viewing was ideal even up to frequency of $2.0 \mathrm{~Hz}$. Using transient stimuli, they showed that viewing a near target caused a response that deviated from one during viewing a far target within $20 \mathrm{~ms}$. Virre 
Han et al., /VOR during near-viewing

et al. proposed that the brain uses canal and otolithic inputs to generate a central estimate of target location in head coordinates, and this is followed by a transformation into ey e movement commands. Snyder and King (1992) confirmed, using transient stimuli that the increased gain associated with near viewing became apparent $\sim 20 \mathrm{~ms}$ after the onset of the stimulus.

In humans, Crane and Demer (1998) applied transient head rotations and identified a gain increase attributable to target distance within 8-18 $\mathrm{ms}$ after the onset of head rotations. Based on the results of gentamicin-induce hair cell lesions of the vestibular laby rinth, it has been suggested that the vergence-mediated component of the response depends on irregular vestibular nerve afferents (Migliaccio et al. 2004). These results imply that Gvor is increased, since visual tracking ey e movements act at much longer latency, even when the near target is head-fixed (Huebner et al. 1992; Johnston and Sharpe 1994; Crane and Demer 1999; Gellman et al. 1990). The EVOR has also been tested in humans with sinusoidal head rotations during viewing of a near target. Reported results are similar to the present study (Figure $2 \mathrm{C}$ and $\mathrm{D}$ ): the gain of compensatory ocular responses is appropriately increased at lower frequencies, but declines with increasing phase shifts as test frequency increases (Hine and Thorn 1987; Crane et al. 1997; Paige et al. 1998). Our results of EVOR while viewing of a distant target during sinusoidal rotation (Figure 2A and B) have also been confirmed: EVOR shows little change in gain or phase shift with increasing frequency up to about $4 \mathrm{~Hz}$ ( Kasteel-van Linge and Maas 1990; Paige et al. 1998). Taken together, these results seem to point to a paradox: responses to transient stimuli indicate an increase of Gvor as the mechanism for increased EVOR gain, but responses to sinusoidal stimulation suggest a non-vestibular contribution that deteriorates at 
Han et al., /VOR during near-viewing

higher frequencies of head rotation.

In the present study, we present evidence that, for human subjects who are oscillated sinusoidally, Gvor is not the main determinant of the increased response. Thus, estimates of Gvor made during viewing of either an earth-fixed or a head-fixed near target were not different in nine out of ten subjects (group medians of 0.98 and 0.97 , respectively). The values of Gvor during near viewing were significantly greater than during viewing of a far, flashing target in darkness (group median 0.91), and the data showed variation (Figure 4). Thus, there ap pears to be a difference on the one hand if Gvor is measured at the onset of a head rotation starting from a stationary position, and on the other hand if Gvor is measured while the head is in motion (sinusoidal oscillations in our study). This difference has been commented on before by Paige et al. (1998), who noted that during fixation of a head-fixed target, if transient stimuli are used, the response gain is decreased to about 0.7 (Huebner et al. 1992) but if sinusoidal stimuli are used, no such gain decreased is apparent (Paige 1994). Paige et al. (1998) go on to speculate that fixation is degraded during high frequency head rotation, with persistent image slip; they predict that if transient stimuli are applied during high-frequency oscillations, that gain would not be decreased, similar to during sinusoidal stimulation -- a result that we confirm in this study.

When we attempted to estimate the contribution of the Gvor to the overall gain of compensatory eye movements, it was typically about $73 \%$, meaning that non-vestibular factors play ed a large role. We consider these factors next. 
Han et al., /VOR during near-viewing

Contributions by non-vestibular mechanisms to generation of compensatory eye movenets

The decline in gain of compensatory eye movements during fixation of an earth-fixed target that occurs at higher frequencies (Figure 2C) suggested that an important non-vestibular contribution was visual tracking Thus, it is well known that when smooth-pursuit eye movements are tested with sinusiodal target motion, gain declines and phase lag increases for stimuli above $1.0 \mathrm{~Hz}$ (Lisberger et al. 1981). At lower frequencies, gain may be close to 1.0 and phase shift almost zero; this behavior is attributed to predictive mechanisms (Dallos and Jones 1963; Bames et al. 2002), which the brain mobilizes to counter delays inherent in the visual sy stem.

In control experiments, we applied two novel approaches to determine if smoothpursuit mechanisms contributed to EVOR. First, we tested compensatory eye movements under strobe illumination, which essentially abolishes retinal slip information (Melvill Jones and $M$ andl 1981), and found that the gain of the response decreased, especially at higher frequencies of head rotation (Figure 6). This gain decrease was also present when we used a strobe flash rate that was a fixed ratio of head rotational frequency, so that target position information was similar throughout. Second we tested compensatory eye movements using sum-of-sines chair rotations, which minimizes the effects of prediction (Figure 7). We found that the tracking gain was decreased during sum-of-sines stimulation compared with gains for rotations at component sine waves, especially those of lower frequency (Figure 7). Since prediction is known to decline at higher frequencies, the difference between sinusoidal and sumof-sines stimulation becomes less obvious for higher component frequencies. Taken together, 
Han et al., NOR during near-viewing

these two results provided circumstantial evidence that smooth-pursuit ey e movements made an important contribution to EVOR during viewing a near target. We then asked the question: Could smooth-pursuit eye movements account for the entire non-vestibular component of the overall resp onse to head rotation?

Inspection of the Bode plots of comp ensat ory ey e movements during viewing an earthfixed near target (Figure 2C and D) and during smooth pursuit (Figure 9A and B) suggests a qualitative difference in the decline of gain and increase of phase shift, with a more abrupt change for smooth pursuit above $1.0 \mathrm{~Hz}$. We attempted to make a more quantitative determination of whether smooth-pursuit could account for all of the non-vestibular component by applying a linear model (Figure 8) and estimating optimal values of nonvestibular parameters at each frequency for each subject during EVOR. We then compared predictions of visual tracking of the non-vestibular part of the model with observed smoothpursuit performance for the same subject and frequency (Figures 9 and 10A). We found that smooth-pursuit performance was inferior to the model's predictions, especially at higher frequencies, when observed pursuit gain was lower and phase lags greater. Interestingly, the model could predict the behavior of EHT during viewing of a near, head-fixed target better than it could predict smooth pursuit (Figure 10A and B).

What mechanism other than smooth pursuit could account for the balance of the nonvestibular contribution? One possibility is a separate "visual fixation" sy stem, with superior tracking properties at higher test frequencies. A body of behavioral and electrophy siolgical work supports such a separate entity (Luebke and Robinson 1988; Ly nch et al. 1977; Leigh 
Han et al., NOR during near-viewing

and Zee 1999), which may depend on directed visual attention to a stationary target. Such sy stems may act at shorter latency than smooth pursuit (Gellman et al. 1990). If this is the case, then could the same fixation mechanism account for the model's ability to predict EHT behavior during viewing of a head-fixed near target (Figure 10B)? Prior studies have demonstrated that the latency to onset of EHT in humans is shorter $(\sim 50 \mathrm{~ms})$ than for smooth pursuit eye movements ( $100 \mathrm{~ms}$ ), which might be an important factor (Carl and Gelman 1987; Huebner et al 1992; Johnston and Sharpe 1994; Crane and Demer 1999). An explanation for this two-fold difference in latency is lacking; however, it seems possible that the brain could hamess vestibular signals, which act at a latency of $<15 \mathrm{~ms}$, to generate eye movements that either track the head-fixed target (EHT) or enhance the VOR (EVOR). Such a suggestion requires electrophy siological confirmation, although recent studies have identified vestibular nucleus neurons with discharge properties that change according to gaze strategies and whether head movements are active or passive (McRea and Luan 2003; Cullen and Roy 2004).

\section{Vergence angle and the compensatory response during near viewing}

During the main experiments, subjects viewed the near visual target monocularly. In this situation, the only stimulus to vergence is accommodation (which is open-loop). We found that vergence angle did not correlate at all with EVOR gain during this testing (Figure $11 \mathrm{~A}$ and B). In control experiments, subjects viewed the targets binocularly, so that vergence angle was appropriate for near viewing. Under these conditions, four of the subjects showed a greater vergence angle, but the gain of EVOR was not increased compared with monocular viewing. In 
Han et al., /VOR during near-viewing

one subject, the vergence angle was similar during monocular and binocular viewing and, in his case, EVOR gain was smaller during binocular viewing. Several prior studies have suggested that vergence angle is an important determinant of EVOR gain. For example, Snyder and King (1992) reported that, for head rotations around an axis ly ing between the otoliths, vergence ange was linearly related to the gain of compensatory movements. However, in a related study, Snyder, Lawrence and King (1992) tested VOR responses during vergence movements, and were able to show that compensatory responses anticipated vergence movements, suggesting that a "central command signal rather than an afferent or efferent copy of vergence position was used to modulate VOR gam". Studies in humans that have employ ed sinusoidal head rotations have either not compared monocular and binocular viewing conditions as we did, or have not directly measured vergence angle (Hine and Thorn 1987). Stronger evidence has been presented to indicate that vergence angle determines response gain during sinusoidal stimulation for the translational VOR in monkeys (Wei et al. 2003; Angelaki 2004). Indeed, vergence may have different effects depending on species (e.g., monkey versus human) or stimulus ty pe (transient rotation from statonary start versus sinusoidal rotation). Based on our present study, we suggest that vergence ange is not the actual determinant of EVOR gain in humans during sinusoidal stimulation and viewing of a near target. Instead, our data indicate that monocular visual cues contain most of the information for appropriate adjustment of gain, although binocular vision of the near tanget may improve EVOR performance. This finding that vergence angle is not correlated with VOR gain during near viewing is consistent with our former study of visually enhanced VOR during mirror viewing(Han et al. 2001). 
Han et al., /VOR during near-viewing

Possible significance of present results for natural behaviors

How revelant are our findings to natural behaviors? During locomotion, head perturbations have a periodicity to them, which is imposed by stepping frequency (Grossman et al. 1988). Fourier analysis of such head movements shows that, in the yaw plane, predominant frequencies ty pically in the range $0.5-2.0 \mathrm{~Hz}$ with harmonic up to $10 \mathrm{~Hz}$. Thus, the range of frequencies employed in the present study are not disimilar to those ocauring during locomotion. Although we may focus our attention on distant objects during walking and running, sometimes it is important to view proximate objects, to avoid collision; under those circumstances EVOR performance during near viewing is important. Our results suggest that, under such circumstance, EVOR is adequate to compensate appropriately for fundamental frequencies, and partially compensates for higher-frequency hamonics. However, some caution is required in extrapolating our results, which concern en bloc rotation to the head-free condition of natural locomotion, since electrophy siolgical studies have identified vestibular nucleus neurons that behave differently during active than passive head rotation (McRea and Luan 2003; Cullen and Roy 2004). Further studies are needed to measure gaze stability, retinal image motion, and visual function during locomotion, as subjects view proximate objects.

\section{ACKNOWLEDGMENTS}

Supported by NIH grant EY06717, NASA/NSBRI NA00208, the Office of Research and Development, Medical Research Service, Department of Veterans Affairs, and the Evenor 
Han et al., /VOR during near-viewing

Armington Fund (to Dr. Leigh). The authors are grateful to Drs. Robert F. Kirsch, David L. Wilson, John D. Porter, William P. Huebner for critical comments. The work reported in this paper constitutes research performed by Yaming $\mathrm{H}$. Han as part of the requirements for her Doctoral Dissertation. 
Han et al., /VOR during near-viewing

Appendix Method for estimating non-vestibular parameters

We developed a superposition model (Figure 8 ) to describe the interactions between vestibular and non-vestibular factors during viewing an earth-fixed near target. The nonvestibular factors include all components that could contribute to EVOR modulation during near viewing such as visual tracking and predictions. The transfer function of the model is specified in equation $\mathrm{Al}$ :

(A1)

In equation A1, Gnvor (intemal gin) and Tnvor (time constant) are parameters representing non-vestibular factors. They are unknown parameters that need to be estimated. Gvor (internal gain) and Tvor (time contant) are parameters representing intemal vestibular factor. They are known parameters: Gvor is measured by apply ing transient perturbations (acceleration $>1,000 \mathrm{deg} / \mathrm{s} / \mathrm{s}$ ) while viewing of an earth-fixed near target during sinusoidal head rotations (paradign 4$).$ Tvor $=15 \sec ($ Cohen et al. 1981$) .{ }^{\wedge}$ is the frequency spectrum. $j=\quad$.

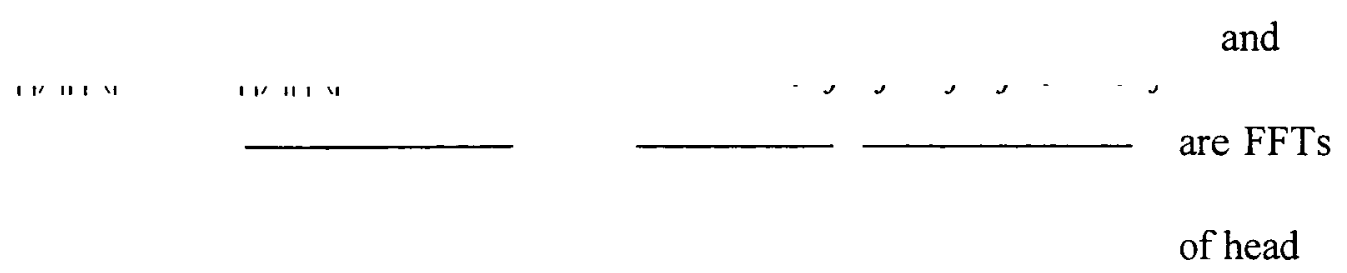

velocity and eye velocity, respectively. is the FFT of eye-in-orbit velocity, adjusted by the equation of Viirre et al. (1986) for near viewing. Thus, the ey e rotation (e.g., right eye, $\left.\theta_{R}\right)$ required to maintain target fixation during head rotations $(\mathrm{H})$ are determined by the radius 
Han et al., NOR during near-viewing

of head rotation $(\mathbf{R})$, the distance of the target from the center of head rotation $(\mathbf{R}+\mathbf{D})$, the interocular distance (I), and the target eccentricity $(\gamma)$, as quantified by the equation A2:

(A2)

For each subject, we measured I (interocular distance) and D (target distance from the subject's viewing ey e) directly.

We then op timized parameters

$\mathrm{R}$ (radius of head rotation) and $\gamma$ (target eccentricity) using equation (A2) to curve fit the EVOR responses at $0.2 \mathrm{~Hz}$ head rotations when subjects were able to continuously foveate on the earth-fixed near target. The optimal parameters for each subject were then used in the equation in the EVOR model ("Viirre's equation" in Figure 8) to account for the retinal image motion induced by head rotation.

To find the optimal values of the non-vestibular parameters Gnvor and Tnwor, we used a nonlinear least square parameter estimation mahod implemented in the frequency domain (Das et al 1998). In the frequency domain, the objective function ${ }^{\circ}(B)$ is defined by equation A3:

where: ${ }^{o}(B)$ is objective function;

$B$ is the unknown parameter vector [Gnvor Tnvor]; $y_{i}$ is the FFTs of the experimental data (EVOR); 
Han et al., /VOR during near-viewing

$f_{i}(B)$ is model outputs given by the transfer function equation (A1);

$N$ is the number of points in FFT;

$i$ is the harmonic number.

Each estimates was based on one trial with a total number of 5,000 data points (corresponding to 10 seconds samplingtime) collected at each frequency during viewing an earth-fixed near target. Prior to running the procedure, noise and saccades were removed interactively. For each estimates, five different starting points were taken in the range of the possible values. The estimation was repeated until opt imization terminated sucessfully. For each subject, we calculated the mean values of the estimations terminated from five initial values. In addition, we evaluated the coefficient of variation (standard deviation / mean value) for each parameter, and the results are shown in Table 1. We then identified the components of non-vestibular factor in two ways: (1) We compared the non-vestibular model predictions of smooth pursuit with the observed performance during near viewing. In this way, we could identify if smooth pursuit could fully account for the non-vestibular componet. (2) We compared the non-vestibular model predictions with the observed EHT performanœ to identify if the same non-vestibular mechanism could account for the model's ability to predict EHT behavior while viewing of a head-fixed near target during head rotations. 
Han et al., NOR during near-viewing

\section{Figure Legends}

Figure 1. General features of EVOR responses from one subject during the main experiments 1-

3. $A$ and $B$ are responses during viewing of the far flashed target in an otherwise dark room.

The gain of the response is close to 1.0 at $0.2 \mathrm{~Hz}$ and 0.9 at $2.0 \mathrm{~Hz}$. C and D are during en-bloc rotation while viewing an earth-fixed near target. At low frequency $(\mathrm{C}, 0.2 \mathrm{~Hz})$, the actual EVOR gain is 1.49 which is close to the ideal gain of 1.50 (calculated from the geometry in Appendix), indicating that the compensatory ey e rotations satisfy the visual demands. At high frequency $(\mathrm{D}, 2.0 \mathrm{~Hz})$, the actual EVOR gain is 1.37 which deviates from the ideal gain 1.50 by $9 \%$ indicating that the ability to compensate for head rotations is impaired. E and $\mathrm{F}$ are during en-bloc rotation while viewing a head-fixed near target (EHT). At low frequency $(\mathrm{E}, 0.2 \mathrm{~Hz})$, the actual EVOR gain is 0.01 which is close to the ideal gain of 0 indicating that VOR is negated. At high frequency $(\mathrm{F}, 2.0 \mathrm{~Hz})$, the actual EVOR gain is 0.39 indicating that VOR is only partially suppressed.

Figure 2. Bode plots summarizing EVOR gain and phase shift, from 10 subjects, during en-bloc rotation during the main experiments $1-3$; head rtations are in the frequency range $0.2-2.8$ Hz.. Plots A and B summarizes responses during viewing the flashed far target in darkness. Note that gain and phase change little over the frequency range tested. Plots C and D are resp onses while viewing an earth-fixed; all subjects showed decreased gain (C) and increased phase shift (D) at high frequencies. For viewing of the head-fixed near target (plots $E$ and $F$ ), tracking gain is plotted with respect to chair stimulus. Subjects showed increased gain (E) and 
Han et al., /VOR during near-viewing

increased phase shift $(F)$ at test frequencies above $1.0 \mathrm{~Hz}$.

Figure 3. Illustration of how Gvor was estimated from head perturbations during sinusoidal rotation. The onset of transient head perturbation is indicated by the vertical arrow in each figure. Peak velocity measurements are marked with asterisks. Plot A shows an example of the perturbation response as the subject views an earth-fixed near target; in plot $B$, he views a head-fixed near target. The change in head and eye velocity (inverted to aid comparisons) is similar during the two visual conditions. For p bt A, Gvor was 1.08; for plot B, Gvor was 1.04.

Figure 4. Histogram summarizing distribution of median Gvor for each of five test frequencies and ten subjects $(n=50)$ when viewing an earth-fixed $(A)$ or a head-fixed $(B)$ near target. Plot $C$ summarizes Gvor measured in darkness. Note that for plots A and B, Gvor values were measured through transient head perturbations but, in plot D, Gvor values are based on steadystate measurements. Plot C summarizes a paired comparison of Gvor during viewing of either an earth-fixed or a head-fixed near target; a paired t-test showed no significant difference ( $p>$ $0.053)$.

Figure 5. Gvor contribution to the overall EVOR response, from 10 subjects, during viewing of an earth-fixed near target at the test frequencies. Gvor contribution is fairly constant at about $73 \%$ of the total behaviors. For those few points that exceed $100 \%$, the value of Gvor exceeded the value of EVOR. 
Han et al., /VOR during near-viewing

Figure 6. Comparisons of EVOR responses, from four subjects, between normal illumination and strobe illumination during viewing of an earth-fixed near target at the test frequency range. Black symbols connected by black line are responses under normal illumination; open sy mbols connected by dotted line are during strobe illumination at $4 \mathrm{~Hz}$ flash rate; gray symbols connected by gray line are during strobe illumination in which the flash rate is 3 times the rotational frequency. All subjects show higher EVOR gain under normal illumination than both strobe ilumination conditions duringhigh frequencies $(\$ 1: 0 \mathrm{~Hz})$ of head rotations.

Figure 7. Comparison of EVOR responses, from four subjects, between sines and sum-of-sines head rotations while viewing an earth-fixed near target. Filled sy mbols connected by solid line are during sinusoidal head rotations; open symbols connected by dotted line are during sum-ofsines rotations. All subjects show higher EVOR gin during sinusoidal versus sum-of-sines stimuli, especially at low frequency components, indicating predictions contribute to EVOR adjustment.

Figure 8. The superposition model used to describe the interaction between vestibular and non-vestibular fact or when viewing of an earth-fixed near target ( ) during en-bloc head rotations in the frequency range $0.2-2.8 \mathrm{~Hz}$. The transfer function is given below the model structure. $G_{n v o r}$ (internal gain) and $T_{n v o r}$ (time constant) are parameters representing nonvestibular factor. They are unknown parameters that need to be estimated. $G_{v o r}$ (internal gain) 
Han et al., NOR during near-viewing

and $\mathrm{T}_{\text {vor }}$ (time contant) are parameters representing internal vestibular sy stem. These are known parameters: $G_{v o r}$ is measured from behaviors in darkness. $T_{v o r}=15 \mathrm{sec}$ (from previous literature). ${ }^{\wedge}$ is the frequency spectrum. $\mathrm{j}=\quad$. is the FFT of eye-in-orbit velocity (adjusted by Viirre's equation for near viewing). and are FFTs of head velocity and eye velocity, respectively. The summing junction on the left is the algebraic summation of (target velocity), (eye velocity) caused by head velocity ), and (the proceeding eye velocity). The result is the retinal error velocity . The summing junction on the right represents the superposition of the internal VOR signal and the internal non-VOR signal calculated by the brain to generate the final eye velocity .

Figure 9. Comparison between smooth pursuit tracking gain predicted by non-VOR model and the measurement from 10 subjects at each of the test frequencies. Results are summarized in Bode plots - gain (A) and phase shift (B). Experimental responses are shown in circles connected by solid line, model predictions are shown in squares connected by dotted line. Model predictions deviated from subjects' responses gain substantially at high frequencies with higher gain and smaller phase shift. Thus, smooth pursuit can not fully account for nonvestibular components that contribute to EVOR responses during viewing of an earth-fixed near target.

Figure 10. Paired comparison of predicted tracking gain for smooth pursuit (A) and EHT during viewing of the near, head-fixed target during chair rotation $(B)$ versus experimentally 
Han et al., NOR during near-viewing

measured near smooth pursuit responses. Each point corresponds to one test frequency for each subject. Data points that fall on the diagonal line correspond to exact predictions of behavior by the model. Note that the model predicts geater tracking gains for smooth pursuit than were observed, but that model predictions are more accurate for EHT beahvior.

Figure 11. Relationship between EVOR gain and vergence angle duringy aw head rotations at $0.2-2.8 \mathrm{~Hz}$. A and B show results during monocular viewing of an earth-fixed target (A) and a head-fixed target (B), from 10 subjects. C shows the comparison of EVOR gains, from 5 subjects, between monocular and binocular viewing, as well as between earth-fixed target viewing and head-fixed target viewing. Note: in $A$ and $B$, the shape of sy mbols codes for frequency; in $\mathrm{C}$, the shape of sy mbols codes for subject. Also in $\mathrm{C}$, open sy mbols are monocular viewing(left part of the vertical dashed line); gray sy mbols are binocular viewing (right part of the vertical dashed line). The vertical dashed line draws the approximate boundary of vergence angle between monocular and binocular viewing. Sy mbols with thick edge (above the horizontal dashed line) are during viewing of an earth-fixed target; sy mbols with thin edge (below the horizontal dashed line) are during viewing of a head-fixed target. Horizontal dashed line shows the app roximate boundary of EVOR gain baween viewing an earth-fixed and a head-fixed target. Note that one subject was able to keep nearly the same vergence angle during monocular and binocular viewing(data endosed by ellipse indicated by arrow), but the EVOR gain during binocular viewing was slightly smaller. 
Han et al., /VOR during near-viewing

\section{REFERENCES}

Angelaki DE. Ey es on target: what neurons must do for the vestibuloocular reflex during linear 
Han et al., /VOR during near-viewing motion. J Neurophysiol 92: 20-35, 2004.

Aw ST, Haslwanter T, Halmagyi GM, Curthoys IS, Yavor RA, AND Todd MJ. Threedimensional vector analy sis of the human vestibuloocular reflex in response to high-acceleration head rotations I. Responses in normal subjects. JNeurophysiol 76: 4009-4020, 1996.

Barnes GR. Visual-vestibular interaction in the control of head and ey e movement: The role of visual feedback and predictive mechanisms. Progress in Neurobiology 41: 435-472, 1993.

Bames GR, Barnes DM, AND Chakraborti SR. Ocular pursuit to repeated, single-cy cle sinusoids reveals behavior compatible with predictive pursuit. J Neurophysiol 84:2340-2355, 2000 .

Barr CC, Schultheis LW, AND Robinson DA. Voluntary, nonvisual control of the human vestibuloocular reflex. Acta Otolaryngol (Stockh) 81: 365-375, 1976.

Biguer B AND Prablanc C. Modulation of the vestibulo-ocular reflex in eye-head orientation as a function of target distance in man. In: Progress in Oculomotor Research, edited by A.F. Fuchs and W. Becker. Amsterdam: Elsevier. p525-530, 1981.

Blakemore C AND Donaghy M. Co-ordination of head and ey es in the gaze changing behavior 
Han et al., /VOR during near-viewing

of cats. J Physiol Lond 300: 317-335, 1980.

Cannon SC, Leigh RJ, Zœ DS, AND Abel L. The effect of the rotational magnification of corrective spectades on the quantitative evaluation of the VOR Acta Otolaryngol (Stockh) 100: $81-88,1985$.

Carl JR AND Gellman RS. Human smooth pursuit: Stimulus-dependent responses. $J$ Neurophysiol 57: 1446-1463, 1987.

Collewijn H AND Smeets JB. Early components of the human vestibulo-ocular response to head rotation: latency and gain. J Neurophysiol 84:376-389, 2000.

Crane BT AND Demer JL. Human gaze stabilization during natural activities: Translation, rotation, magnification, and target distance effects. J Neurophysiol 78:2129-2144, 1997.

Crane BT, Vïrre ES, AND Demer Л. The human horizontal vestibulo-ocular reflex during combined linear and angular acceleration. Exp Brain Res 114: 304-320, 1997.

Crane BT AND Demer JL. Human horizontal vestibulo-ocular reflex initiation: effects of acceleration, target distanœ, and unilateral deafferentation. J Neurophysiol 80: 1151-1166, 1998. 
Han et al., /VOR during near-viewing

Crane BT AND Demer JL. Latency of voluntary cancellation of the human vestibulo-ocular reflex during transient y aw rotation. Exp Brain Res 127: 67-74, 1999.

Camenter RHS. The visual origins of $\propto$ cular motility. In: Vision and Visual Function. Vol 8. Eye Movements. Edited by J.R. Cronly-Dillon. MacMillan Press, London p 1-10, 1991.

Cohen B, Henn V, Raphan T, AND Dennett D. Velocity storage, ny stagmus, and visualvestibular interactions in humans. Ann NY Acad Sci 374: 421-433, 1981.

Cullen KE, Belton T, AND MoCrea RA. A non-visual mechanism for voluntary cancellation of the vestibulo-ocular reflex. Exp Brain Res 83: 237-252, 1991.

Cullen KE AND Roy JE. Signal processing in the vestibular sy stem during active versus passive head movements. J Neurophysiol 91: 1919-1933, 2004.

Dallos PJ AND Jones RW. Learning behavior of the eye fixation control sy stem. IEEE Trans Automat Control AC-8: 218-227, 1963.

Das VE, Leigh RJ, Thomas CW, Averbuch-Helle L, Zivotofsky AZ, DiScenna AO, AND Dell'Osso LF. Modulation of high-frequency vestibulo-ocular reflex during visual tracking in 
Han et al., NOR during near-viewing humans. J Neurophysiol 74: 624-632, $1995 \mathrm{a}$.

Das VE, Zivotofsky AZ, DiScenna AO, AND Leigh RJ. Head perturbations during walking while viewing a head-fixed target. Aviation, Space and Environmental Medicine 66: 728-732, 1995b.

Das VE, DiSœnna AO, Feltz A, Yaniglos SS, AND Leigh RJ. Tests of a linear model of visualvestibular interaction using the technique of parameter estimation. Biol Cybern 78: 183-195, 1998.

Das VE, Yaniglos SS, AND Leigh RJ. The influenœ of light on modulation of the human vestibulo-ocular reflex $J$ Vestibular Res 10:51-55, 2000.

Gellman RS, Carl JR, AND Miles FA. Short btency ocular-following responses in man Vis Neurosci 5: 107-122, 1990.

Grossman GE, Leigh RJ, Abel LA, Lanska DJ AND Thurston SE. Frequency and velocity of rotational head perturbations during locomotion. Exp Brain Res 70: 470-476, 1988.

Grossman GE, Leigh RJ, Bruce EN, Lanska DJ AND Huebner WP. Human vestibulo-ocular reflex during locomotion. $J$ Neurophysiol 62: 264272, 1989. 
Han et al., NOR during near-viewing

Guild SJ, Austin PC, Navakatiky an M, Ringwood JV AND Malpas SC. Dynamic relationship between sympathetic nerve activity and renal blood flow: a frequency domain approach. $A m J$ Physiol Regul Integr Comp Physiol 281(1):R206-212, 2001.

Han Y, Somers JT, Kim J-I, Kumar AN, AND Leigh RJ. Ocular responses to head rotation during mirror-viewing. J Neurophysiol 86:2323-2329, 2001.

Han Y, Kumar AN, AND Leigh RJ. Test of a superposition model of the interaction between vestibular and vergence sy stems using a parameter estimation technique. Soc Neurosci Abstr 411.11, 2004.

Han Y, Kumar AN, Somers JT, Reschke MF, AND Leigh RJ. Effects of retinal image slip on modulation of visual-vestibulo-ocular reflex during near-viewing Ann N Y Acad Sci, 2005

Hine T AND Thorn F Compensatory eye movements during active head rotation for near targets: effects of imagination, rapid head oscillation and vergence. Vision Res 27(9): 1639$1657,1987$.

Huebner WP, Leigh RJ, Seidman SH, Thomas CW, Billian C, DiScenna AO, AND Dell'Osso LF. Experimental tests of a superposition hy pothesis to explain the relationship between the 
Han et al., NOR during near-viewing

vestibuloocular reflex and smooth pursuit during horizontal combined ey e-head tracking in humans. J Neurophysiol 68: 1775-1792, 1992.

J.C. Living without a balancing mechanism. New Engl J Med 246: 458-460, 1952.

Johnston JL AND Sharpe JA. The initial vestibulo-ocular reflex and its visual enhancement and cancellation in humans. Exp Brain Res 99: 302-308, 1994.

Kasteel-van Linge A AND Maas AJJ. Quantification of visuo-vestibular interaction up to 5.0 $\mathrm{Hz}$ in normal subjects. Acta Otolaryngol (Stockh) 110: 18-24, 1990.

Krauzlis RJ AND Lisberger S.G. A control systems model of visually-guided smooth pursuit ey e movements based on behavioral observations. J Comp Neurosci 1:265-283, 1994.

Leigh RJ AND Zee DS. The neurology of eye movements. New York: Oxford University Press, 1999.

Lisberger SG, Evinger C, Johanson GW, AND Fuchs AF. Relationship between ey e acceleration and ret inal image velocity during foveal smooth pursuit in man and monkey. $J$ Neurophysiol 46: 229-249, 1981. 
Han et al., /VOR during near-viewing

Luebke AE AND Robinson DA. Transition dy namics between pursuit and fixation suggest different sy stems. Vision Res 28:941-946, 1988.

Lynch JC, Mountcastle VB, Talbot WH, AND Yin TCT. Parietal lobe mechanisms for directed visual attention. $J$ Neurophysiol 40:362-389, 1977.

Maas EF, Huebner WP, Seidman SH AND Leigh RJ. Behavior of human horizontal vest ibuloocular reflex in response to high-acceleration stimuli. Brain Res 499: 153-156, 1989.

McCrea RA AND Luan H. Signal processing of semicircular canal and otolith signals in the vestibular nuclei during passive and active head movements. Ann NY Acad Sci 1004: 169-182, 2003.

Melvill Jones G AND Mandl G. Motion sickness due to vision reversal: Its absence in stroboscopic light. Ann NY Acad Sci 374: 303-311, 1981.

Migiaccio AA, Minor LB, AND Carey JP. Vergenœ-mediated modulation of the human vestibulo-ocular reflex is eliminated by a partial peripheral gentamicin lesion. Exp Brain Res, 2004.

Paige GD. Senescence of human visual-vestibular interactions: smooth pursuit, op tokinetic, 
Han et al., NOR during near-viewing

and vestibular control of eye movements with aging. Exp Brain Res 98:355-72, 1994.

Paige GD, Telford L, Seidman SH, AND Barnes GR. Human vestibuloocular reflex and its interactions with vision and fixation distance during linear and anglar head movement. $J$ Neurophysiol 80:2391-2404, 1998.

Ramat S, Somers JT, Das VE, AND Leigh RJ. Conjugate ocular ascillations during shifts of the direction and depth of visual fixation. Invest Ophthalmol Vis Sci 40: 1681-1686, 1999.

Snyder LH AND King WM. Effect of viewing distance and the location of the axis of rotation on the monkey's vestibulo-ocular reflex (VOR). I. Ey e movement responses. J Neurophysiol $67: 861-874,1992$.

Snyder LH, Lawrence DW, AND King WM. Changes in vestibulo-ocular reflex(VOR) anticip ate changes in vergence angle in monkey. Vision Res 32:569-575, 1992.

Virre E, Tweed D, Milner K, AND Vilis T. A reexamination of the gain of the vestibuloocular reflex. J Neurophysiol 56: 439-450, 1986.

Wei M, DeAngelis DC, AND Angelaki DE. Do visual cues contribute to the neural estimate of viewing distance used by the oculomotor sy stem? J Neurosci 23:8340-8350, 2003. 